\title{
La transformación de Santiago: un caso frustrado de intervención urbana a gran escala $(1872-1929)^{1}$
}

Katherine Vyhmeister-Fábregas. Universidad Adolfo Ibáñez, Santiago, Chile.

RESUMEN | Una primera discusión respecto a la necesidad de intervenir la ciudad de Santiago a gran escala se instaura en la esfera pública a partir de la década de 1870, y se extiende hasta las primeras décadas del siglo xx. Sin embargo, y a pesar de la gran cantidad de años instalada en el debate público, esta anhelada transformación no se logrará llevar a cabo. El presente artículo explora los diferentes factores que impidieron realizar esta intervención, en una época en que las autoridades buscaban insertar el país en el mundo moderno. Se logrará arrojar ciertas luces sobre este caso a partir de las discusiones en el Congreso Nacional, instancia más cercana a la aprobación y realización concreta de alguna propuesta para transformar la ciudad.

PALABRAS CLAVE | historia urbana, planificación urbana, renovación urbana.

ABSTRACT Between the 1870's and the first decades of the Twentieth Century, a discussion about the need of a large-scale urban intervention in Santiago de Chile appeared in the public sphere. However, despite all the years in which this transformation discussion was installed in the public debate, the idea was never carried out. This paper examines the different factors that prevented this intervention to be accomplished, in a moment in which the authorities tried to position the country as part of the modern world. For this purpose, this study will analyze the discussions that took place in the National Congress, setting in which a large-scale proposal could be approved.

KEYWORDS | urban history, urban planning, urban renewal.

Recibido el 2 de marzo de 2017, aprobado el 8 de noviembre de 2017

E-mail: kvyhmeister@uai.cl

$1 \longdiv { \text { Este artículo es producto de la investigación realizada en el marco de la tesis de magíster titulada } }$ Discutir la ciudad. Tres proyectos de transformación de Santiago en el Congreso Nacional 1872-1930, defendida en noviembre de 2014, para optar al grado de Magíster en Historia por la Universidad Adolfo Ibáńez, Chile. La autora agradece las valiosas observaciones de los evaluadores. 


\section{Introducción}

Para mediados del siglo XIX, las repúblicas latinoamericanas se encontraban abocadas a la construcción de sus estados nacionales. Desde que los procesos independentistas se consolidaron, surgió el desafío de idear un proyecto de país, que fue encauzado a partir de las visiones y anhelos de las clases dirigentes, que encontraron en las naciones europeas modernas, como Francia o Gran Bretańa, un modelo al cual seguir (Collier \& Sater, 2005; Romero, 2014). En este sentido, y no sin dificultades y contradicciones, la construcción de identidad fue parte constitutiva de la trayectoria latinoamericana hacia la modernidad. Y si bien este nuevo paradigma político, social, cultural y económico surge en la Europa occidental, será rápidamente incorporado, adaptado y recontextualizado en el Nuevo Continente (Larraín, 1997).

En este marco, y particularmente respecto a los cambios de modelos que la modernidad traerá consigo, las élites urbanas desempeñarán un rol fundamental, puesto que tanto en Europa, durante la segunda Revolución Industrial (Levin, Forgan, Hessler, Kargon \& Low, 2010), como en América Latina, durante las construcciones nacionales (Almandoz, 2013; Romero, 2014), se harán cargo de liderar importantes procesos que tendrán como principal objetivo el ideal de progreso. De este modo, las ciudades latinoamericanas, particularmente sus capitales, se articularon como escenarios fundamentales para demostrar su participación en el mundo moderno europeo (Gorelik, 2003), permitiendo a las élites gobernantes concretar de manera visual el proyecto nacional que intentaban impulsar $y$, a la vez, dar cuenta del estado de progreso que se había alcanzado con los años (Almandoz, 2010; Vicuña, 1996).

Ya desde el siglo xviI en París se había concebido la ciudad "moderna", diseñada para atraer la atención de los residentes y visitantes a partir de nuevas experiencias urbanas, como la construcción de llamativas residencias e infraestructura urbana sin precedentes, que orientaban la ciudad hacia el futuro (DeJean, 2014). Este concepto de ciudad moderna será ampliamente desarrollado durante la transformación de París entre 1853 y 1870, encargada por el emperador Napoleón III a GeorgesEugène Haussmann, y que se establecerá como un hito inaugural de la modernidad urbana (Harvey, 2003), replicada en otras capitales y ciudades del mundo.

La civilización, el embellecimiento, el progreso y la higiene, entre otros, fueron algunos de los valores modernos con los que las élites latinoamericanas se identificaron (Almandoz, 2013). Fue precisamente en torno a estos ejes que durante la segunda mitad del siglo xix se inició en Santiago un periodo de reflexión sobre el estado de la ciudad y la necesidad de remodelarla, con miras a generar una proyección urbana adecuada que dejara atrás la ciudad colonial ${ }^{2}$ (Hofer, 2003). Esta discusión sobre los problemas presentes en ella y el mejor método para darles solución, adscribiendo a las ideas sobre planificación urbana que tanto en Estados Unidos como en Europa se estaban desarrollando (Giedion, 2009; Gravagnuolo, 2010), se

No obstante, la cuadrícula colonial se establecerá como la estructura urbana articuladora del centro de la ciudad aun durante el siglo xx (Gross, 1990; Rosas, 1985). 
proyectó hasta las primeras décadas del siglo $\mathrm{xx}$, dando cuenta de su gran persistencia en el interés público.

Entre los diferentes espacios en que se produjo el debate en Chile, las sesiones del Congreso Nacional se establecieron como instancias fundamentales para pensar y discutir sobre la ciudad. Los debates se llevaron a cabo tanto en la Cámara de Diputados como en el Senado, enviándose distintos proyectos de una Cámara a otra con los reparos y correcciones correspondientes. Adicionalmente, las propuestas eran enviadas a comisiones que se formaban para evaluarlas y que debían emitir informes con conclusiones que permitieran a los parlamentarios tomar una decisión sobre el asunto correspondiente (Valdés, 1906). Por lo tanto, eran diversas las instancias por las que atravesaban los proyectos, generando diferentes espacios de debate, lo que a su vez podía dificultar una resolución expedita (Gross, 1990).

En definitiva, el Congreso fue la instancia más cercana a la realización concreta de alguna propuesta para transformar la ciudad, debido a que la única manera en que esta pudiera ser intervenida a gran escala era mediante la aprobación de un proyecto de ley. Entre 1872 y 1929 es posible identificar tres grandes momentos de discusión en torno a la necesidad de generar cambios profundos en Santiago, a partir de los cuales se definirán diversas problemáticas que los trascienden y que no lograrán resolverse durante el periodo en estudio ${ }^{3}$. El primero se desarrolló entre 1872 y 1874; el segundo, entre 1892 y 1909; y el tercero, entre 1910 y 1929 . De estos tres periodos, dos se concretaron en la aprobación de leyes, una en 1874 y la otra en 1909, las cuales, sin embargo, no tendrán las consecuencias esperadas.

Las propuestas de intervención presentadas en los proyectos de transformación de Santiago, sobre todo los planos que los acompańaban, han sido particularmente abordadas por la historiografía en las últimas décadas (Aguirre \& Castillo, 2004; Fernández, 2014; Fuentes, 2009; Gross, 1989, 1990, 1995; Gurovich, 2000, 2003; Hidalgo \& Camus, 2006; Martínez, 2006, 2007a, 2007b; Strabucchi, Vicuña, Hidalgo \& Rosas, 2013). Si bien se ha establecido que, en su gran mayoría, los proyectos discutidos en el Congreso Nacional no se aprobaron debido tanto a factores económicos como a desacuerdos políticos e intereses particulares, aún quedan asuntos pendientes por analizar respecto de las causas que frustraron una intervención a gran escala de la capital, a pesar de contarse con dos leyes aprobadas.

Considerando el contexto parlamentario particular que vio surgir los distintos proyectos de modernización urbana, el presente trabajo busca identificar los factores que, de una u otra forma, contribuyeron a que Santiago no pudiese experimentar entre 1872 y 1929 una transformación profunda, que sí estaba teniendo lugar en otras capitales latinoamericanas, como Buenos Aires o Río de Janeiro. Se propone como punto de partida el análisis de factores propios tanto de las discusiones parlamentarias y de la administración municipal, como del contexto histórico. Tal enfoque permite concluir que a pesar del gran interés que generó la idea de una renovación urbana de la capital que apuntara hacia la modernización de la ciudad, y 
del éxito aparente que se demostró a partir de la promulgación de las leyes de 1874 y 1909, fueron precisamente las consecuencias negativas de estas leyes para el desarrollo urbano de Santiago las que en definitiva impidieron concretar, de la manera en que se había proyectado, una transformación urbana a gran escala durante las décadas finales del siglo xIX y las primeras del siglo xx.

\section{La necesidad de transformar la ciudad, 1872-1874}

El primer proyecto que contempló una intervención "global" de la capital surgió hacia 1872 en el Senado, a partir de la discusión de una ley para realizar el levantamiento de un plano de Santiago ${ }^{4}$. En el marco de este proceso legislativo, la Comisión de Gobierno y Relaciones Exteriores redactó un proyecto sobre "las grandes e imperiosas mejoras que para consultar su salubridad, su ornato i su existencia misma exije la capital de la República [sic]" (Cámara de Senadores, 24ª Sesión Ordinaria [c.s., s.o.], 1872, p. 117). A partir de este documento comenzarán a ser discutidas en el Congreso las opciones y posibilidades de una intervención a gran escala en la ciudad, puesto que se comenzarán a evaluar asuntos como los límites urbanos, apertura de calles y proyectos de infraestructura como alcantarillado o la canalización del río Mapocho.

No obstante tal impulso, la iniciativa de realizar algunas mejoras a la capital no sería fácil de concretar, especialmente a causa de la deplorable situación económica por la que desde hacía décadas atravesaba la Municipalidad de Santiago (Fernández, 2014; Hidalgo \& Sánchez, 2006), y que le impedía hacerse cargo de los servicios básicos que debía prestar a los habitantes de la ciudad, como el alumbrado o la policía de seguridad. El gran problema que por décadas sufrió el Municipio fue que los gastos que realizaba eran mayores que las entradas recibidas por las contribuciones que se cobraba a los vecinos. En consecuencia, cada año debía endeudarse para poder cumplir con la administración de la ciudad, obligando al Estado a hacerse cargo de algunos aspectos como el de policía y guardia del presidio urbano (Cámara de Diputados, 36a Sesión Extraordinaria [C.D., s.E.], 1877).

A pesar de que las intervenciones urbanas solo eran posibles a partir de un proyecto de ley aprobado en el Congreso, los eventuales gastos que ellas implicaban recaían en la Municipalidad, órgano gubernamental directamente a cargo de la administración de la ciudad. Por lo tanto, si ya para el Municipio era difícil sostener los costos de los servicios básicos, pensar en financiar la compra de los terrenos, las demoliciones y construcciones propuestas, parecía estar fuera de alcance. No obstante, y a pesar de la escasez de recursos para destinar a una propuesta como esa, durante 1873 y parte de 1874 el proyecto siguió discutiéndose y siendo enviado de una Cámara a otra, con las respectivas modificaciones.

Los principales argumentos de aquellos que defendían la renovación urbana se relacionaban con la higiene, salubridad, ornato, embellecimiento, facilidad

José Miguel de la Barra, intendente de Santiago entre 1843 y 1849, intentó llevar adelante un amplio plan de reformas en la capital. No obstante, su propuesta no tuvo el impacto y alcances similares a la discusión sobre la transformación de Santiago desarrollada a partir de 1870 (Hidalgo \& Camus, 2006; Hidalgo \& Sánchez, 2006). 
de tráfico y previsión ante temblores e incendios. Particularmente la higiene y la salud pública se establecieron como temas relevantes en la agenda pública durante las últimas décadas del siglo xix y las primeras del $\mathrm{xx}$, en especial a partir de los brotes de epidemias que azotaban a la población periódicamente (Ibarra, 2016). Tal fue el caso de la capital en los primeros años de la década de 1870, en que las epidemias fueron una de las razones fundamentales para impulsar cambios en su estructura (Folchi, 2007). El senador Vicuña expresaba hacia 1872 que los mejores datos hablaban de 8000 muertos y que las malas condiciones en que vivían algunos sectores de la sociedad propiciaban el contagio de enfermedades: "(...) el pobre ha sido únicamente víctima de la epidemia, porque no pasan [de] doce las personas decentes que han muerto por esa causa, i esto debido a la mala alimentación i al desaseo en que vive nuestra jente del pueblo [sic]" (c.s., 25a s.o., 1872, p. 125). Ante esta situación de precariedad de parte importante de la población, el Senador juzgaba necesaria una transformación de la ciudad, para lo cual el Congreso debía asignarle más fondos a la Municipalidad de Santiago. Esto último era fundamental si se pretendía llevar adelante las obras.

Argumentos como los señalados apoyaban fuertemente no solo el ensanche y apertura de calles, sino también la regulación de la construcción de ranchos y conventillos, en los que sus ocupantes vivían en estado de hacinamiento (Hidalgo, 2002). El hecho de que se dejara a los privados la creación de nuevas poblaciones en las zonas periféricas de la ciudad generó grandes problemas al Municipio de Santiago durante la segunda mitad del siglo xix, puesto que los dueńos de chacras cercanas a los límites urbanos realizaban parcelaciones de sus terrenos, construyendo en ellas edificaciones precarias para luego venderlas al Municipio o arrendarlas (Valdés Cange, 1910). En consecuencia, los habitantes de estos sectores exigían a la Municipalidad servicios básicos como alcantarillado, alumbrado y policía de seguridad, debiendo dicha corporación hacerse cargo de un crecimiento urbano sin planificación alguna, definido solo por los propietarios de aquellos terrenos. Por esta razón, algunos parlamentarios se negarían, más adelante, a la ampliación de los límites urbanos de la ciudad (De Ramón, 1985).

A pesar de las necesidades y beneficios aparentes de realizar una transformación de la ciudad, que cada vez albergaba a un mayor número de habitantes, hubo quienes se opusieron rotundamente a ella. Uno de los aspectos que mayor debate generó fue la delegación a la Municipalidad de ciertas atribuciones que hasta ese momento eran propias del Congreso, con lo que se buscaba una mayor eficiencia en la administración de la ciudad. El principal recelo de los parlamentarios estuvo en resguardar la facultad de decidir sobre los trazados, ensanches y expropiaciones de terrenos (Gross, 1990). Por otra parte, la conocida y deficiente situación económica del Municipio llevó a que algunos parlamentarios simplemente prefirieran discutir otros proyectos que sí tuvieran reales posibilidades de concretarse o, al menos, de obtener gran parte de los resultados propuestos. El senador Vial, por ejemplo, opinaba que el proyecto debía acotarse a lo estrictamente necesario, como el ensanche de un par de calles o la realización del Camino de Cintura (c.s., 11 a s.o., 1873), que permitiría contar con una avenida de circunvalación que hiciera 
más expedito el tránsito por la ciudad y a la vez delimitara el sector céntrico de las zonas periféricas (Pérez \& Rosas, 2010).

Estas discusiones sobre la ciudad se insertaron en momentos en que prácticamente todos los ámbitos de la construcción de la nación se encontraban en desarrollo. Para la década de 1870 se estaba debatiendo sobre importantes proyectos, como la Reforma Electoral, Reforma al Código Penal, Reforma Constitucional y a la Instrucción Pública. Aquello podría llevar a comprender por qué para algunos parlamentarios realizar cambios en la estructura urbana de Santiago no parecía una necesidad de primer orden. A esto último se sumaba el rechazo al evidente centralismo de la propuesta, especialmente a los privilegios que se estaban otorgando a la capital en desmedro de otras ciudades del país, críticas que estarán presentes en el debate durante todo el periodo en estudio.

Cabe destacar que a pesar de los argumentos desfavorables al proyecto, este logró aprobarse en ambas Cámaras y ser publicado en junio de 1874 con algunas variaciones a la propuesta inicial, bajo el título "Apertura i prolongacion de calles i paseos públicos en la ciudad de Santiago". La modificación de algunos artículos da cuenta de un intento por acotar el proyecto para que los fondos municipales no se vieran afectados de sobremanera, por lo que solo se incluyeron las reformas más urgentes, como, por ejemplo, la regulación de líneas y alturas de construcción. La importancia de esta ley radica en que es posible considerarla como lo que en la actualidad se conoce como un plan regulador de la ciudad. No solo proveía de soluciones urbanas para esos momentos, sino que también preveía una planificación futura, estableciendo las guías a las que debían apuntar las diferentes intervenciones (León, 1975). Para este fin, Benjamín Vicuña Mackenna (1873), en su cargo de intendente de la Provincia de Santiago, encargó al ingeniero Ernest Ansart la creación de un plano de la ciudad en el que se plasmaran estas directrices (figura 1).

Si bien se puede proponer la discusión de este proyecto como exitosa, pues se concretó en la promulgación de una ley, sus consecuencias negativas a nivel urbano comenzaron a evidenciarse unos ańos más tarde. La mayor dificultad se relacionó con el incumplimiento de la ley por parte de propietarios y autoridades correspondientes, al no existir sanciones asociadas a esta falta. En este sentido, algunas disposiciones, sobre todo las que normaban las líneas y alturas de construcción, fueron ignoradas por algunos propietarios de terrenos y, como consecuencia, se generó una situación contraria al objetivo del proyecto, que buscaba establecer cierta armonía y homogeneidad en los edificios y calles de la ciudad. Así, la situación de Santiago siguió siendo prácticamente la misma durante los siguientes años, frustrándose la esperada transformación, impulsada en este periodo en gran medida por el intendente Benjamín Vicuña Mackenna.

Fue recién en 1892 cuando comenzó a desarrollarse una nueva discusión, que buscó establecer posibles soluciones y garantizar el cumplimiento de la ley. Este largo lapso de tiempo transcurrido se puede comprender si se consideran algunos acontecimientos como la Guerra del Pacífico, desarrollada entre 1879 y 1883 contra Perú y Bolivia, y el posterior ejercicio de soberanía tras la victoria. Las consecuencias económicas positivas de la anexión de Tarapacá y Atacama se hicieron evidentes en un gran impulso a las obras públicas, infraestructura y conectividad del país, al 
igual que a su industrialización, lo que llevó a la creación del Ministerio de Obras Públicas en 1887 (Collier \& Sater, 1998; Ortega, 2010).

FIGURA I | Plano de Santiago (1875) por Ernest Ansart

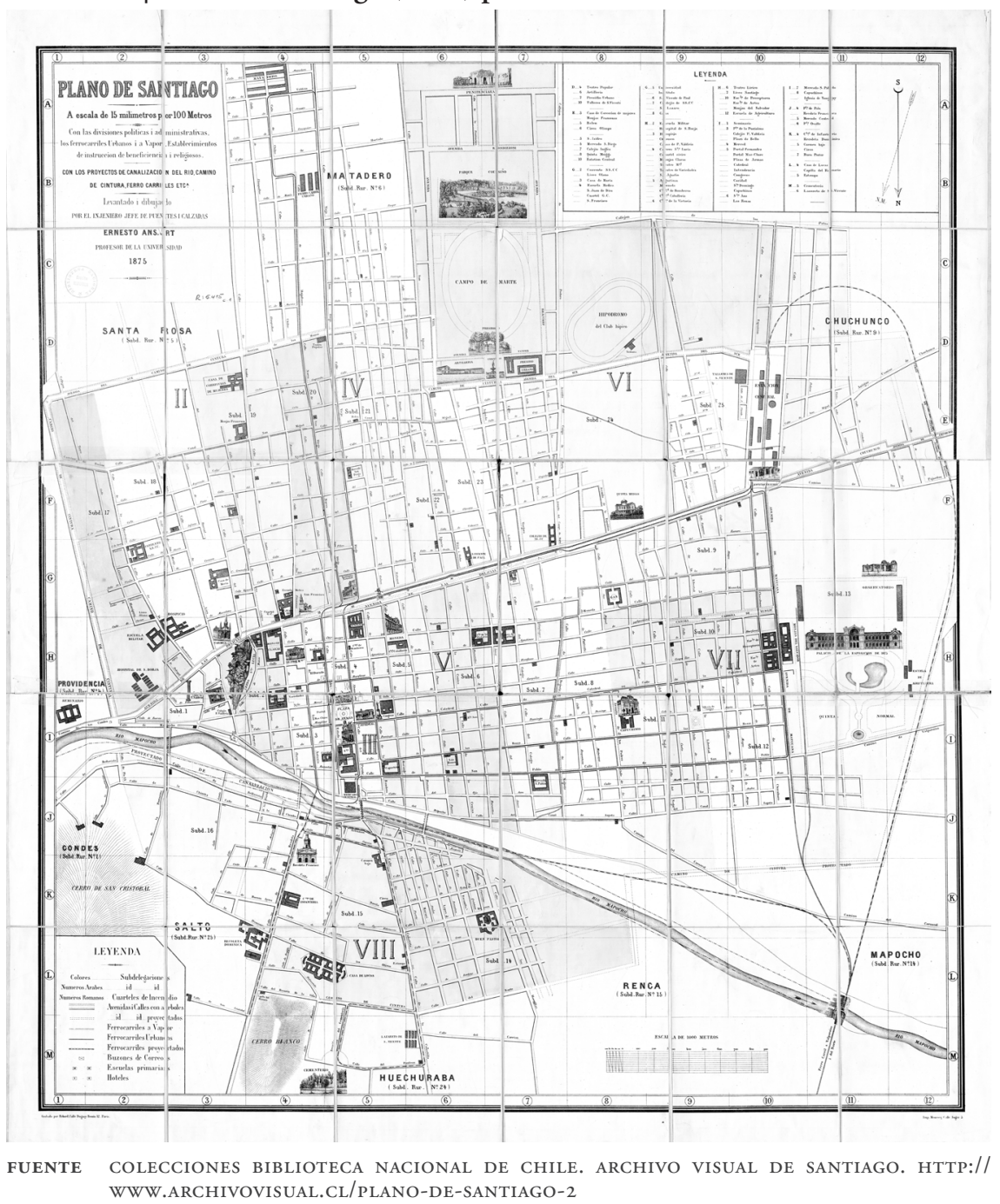

Por otro lado, entre 1881 y 1888 se resolvieron las disputas limítrofes con Argentina (1881), la Ocupación de la Araucanía (1883) y la incorporación de la Isla de Pascua al territorio nacional (1888). La década de 1880 estuvo marcada, de este modo, por acontecimientos relevantes en la configuración territorial. Los sucesos políticos en torno a la guerra civil de 1891 también postergaron la posibilidad de búsqueda de alguna solución a la situación de la ciudad (Carvajal, 1929a). Una vez superada la 
contingencia nacional, que marcó la preocupación de las autoridades por estos años, se hizo posible volver a enfocar la discusión en el estado y proyección de la capital.

\section{Los problemas continúan sin resolverse, 1892-1909}

En 1892, los parlamentarios comenzaron a discutir un nuevo proyecto de transformación de Santiago que buscaba salvar las dificultades que la ley de 1874 había generado. Ya en 1888, el diputado Valentín Letelier denunciaba que no se podía citar un solo caso en que el objetivo de la ley se hubiese cumplido: "Lo único que se ha conseguido es, por una parte, estorbar el desarrollo normal de la ciudad, i, por otra, dar nacimiento en el barrio central a una arquitectura irregular i sin gusto, que afecta sobremanera el aspecto de nuestros edificios [sic]" (Cámara de Diputados,

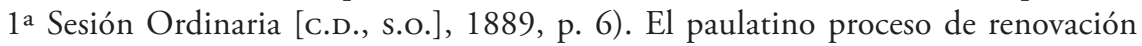
arquitectónica en el que nuevas edificaciones, algunas de ellas siguiendo y otras ignorando las líneas y disposiciones de construcción de 1874, comenzaron a convivir con aquellas más antiguas que demorarían varias décadas en ser reemplazadas, dificultó alcanzar el objetivo del proyecto de transformación.

Resulta necesario destacar que, en este segundo periodo de discusión, fue la Municipalidad de Santiago la institución que impulsó en gran medida el debate orientado a establecer una normativa urbana apropiada para la capital. El informe de la Comisión de Gobierno y Relaciones Exteriores reflejó una búsqueda de regular, mediante la aprobación previa de un plano de la ciudad por parte de la Municipalidad, la construcción y reconstrucción de las edificaciones; el ancho de las calles; la altura de los edificios; las indemnizaciones por las expropiaciones; las condiciones en que deberían edificarse o reconstruirse los edificios que se situaban en los costados de las calles, plazas y otros bienes nacionales de uso público; y las penas por infracciones a la ley (C.D., 21 a s.O., 1892). Resulta evidente que el punto sobre el establecimiento de penas por incumplimiento de la ley surgió a partir de la experiencia de los años anteriores, en que algunos propietarios simplemente omitían la normativa al no existir ninguna sanción determinada por ley, estableciéndose tal situación como uno de los problemas fundamentales legados desde 1874.

Los meses pasaron y la discusión pareció no avanzar mayormente en las cámaras del Congreso Nacional. Ante esta situación de escaso interés, se llegó a la propuesta de dictar leyes generales de transformación de ciudades y que cada localidad las adaptara a sus propias posibilidades. Sin embargo, la diferencia de tamaño y presupuesto de las distintas localidades del país era problemática para la creación de leyes generales; y, sobre todo, el rol de capital que debía encarnar la ciudad de Santiago la hacía situarse en un rango superior al resto de las ciudades del país. El diputado Paulino Alfonso realizó una enfática intervención en la que expuso que en la capital se hacía indispensable la creación de "parques i anchas calles o avenidas que serian tal vez inconvenientes en otros pueblos [sic]" (C.D., 8a s.E., 1894, p. 135). De esta manera, la capital requería de ciertas implementaciones que la establecieran como el fiel reflejo del estado de progreso de la nación (Mackenna, 1915).

Los últimos años del siglo xix presentaron una situación favorable para llevar adelante una renovación de la ciudad. Los edificios de adobe existentes en esos 
momentos no eran de gran valor y aún había poco tráfico de vehículos (c.D., 20a S.E., 1894), por lo que se daban las condiciones adecuadas para realizar intervenciones importantes a un costo razonable. En este periodo de discusión, además de las dificultades económicas, se observa que el mayor obstáculo que tuvo que enfrentar el proyecto fue el tiempo transcurrido desde el primer informe de la Comisión de Gobierno, debido a que para los meses finales de 1894 la conformación del Congreso Nacional había cambiado. Para ese año solo formaban parte de la Cámara dos de los miembros de aquella Comisión informante, lo que impedía contar con una mayor explicación sobre las modificaciones propuestas. En este contexto, la posibilidad de votar el proyecto de manera informada y acertada se dificultaba, lo que se comprueba en las palabras del diputado Raimundo Silva Cruz:

Hai un plano, que no conocemos; hai un informe de una Comision que ya no existe; i con estas condiciones la Cámara va a decidir: se aprueba el plano segun el cual deberán abrirse esta i la otra avenida, formarse tales plazas, ensancharse cuales calles. Por su parte, la Comision nos agrega: yo estimo que debe aceptarse la apertura de tal avenida i suprimirse tal otra. ¿Qué antecedentes tenemos para resolver con un conocimiento de causa siquiera relativo? Ninguno [sic] (c.D., 11 a S.E., 1894, p. 178).

En consecuencia, el escenario al cual se enfrentaban los diputados para proseguir con la discusión se tornaba cada vez más complejo, mientras el contexto económico se mostraba poco propicio. Las políticas económicas chilenas de finales del siglo XIX parecieron haber profundizado los problemas en este ámbito, al terminar con la inconvertibilidad monetaria entre los años 1895 y 1898, hecho que habría servido solo para acentuar la depresión (De Ramón, 1985). Otro aspecto relevante es la instauración, a partir de 1891, del régimen de gobierno comúnmente denominado como "parlamentarismo", que implicó la disminución del poder presidencial y el fortalecimiento del Congreso en su capacidad de legislar y gobernar, lo que generó grandes inconvenientes para realizar y dar continuidad a los programas de gobierno, agudizados por la gran rotativa ministerial. Esta práctica fue reflejo de las amplias facultades que alcanzaron los legisladores, puesto que podían interpelar y destituir a los ministros de Estado sin que el Presidente de la República tuviera posibilidad de intervenir (Gazmuri, 2012; Heise, 1982).

No obstante, el proyecto de transformación de Santiago siguió su curso y tras ser aprobado en la Cámara de Diputados en 1894, junto a un plano que lo acompañaba confeccionado por el director de Obras Municipales, el ingeniero Manuel Concha (figura 2), pasó al Senado para ser discutido y sancionado. En el marco de este proceso, la Municipalidad de Santiago ratificó en noviembre del año siguiente un informe relativo al proyecto redactado por el ya mencionado ingeniero Concha. Resulta interesante dar cuenta de la estrategia empleada por el Municipio al aprobar este informe, puesto que fue encargado en clara respuesta al informe redactado por el Director General de Obras Públicas, Alejandro Bertrand, el 28 de octubre del mismo año. 
Figura 2 | Plano de Santiago. Proyecto de ensanche de calles y avenidas diagonales (1894) por Manuel Concha

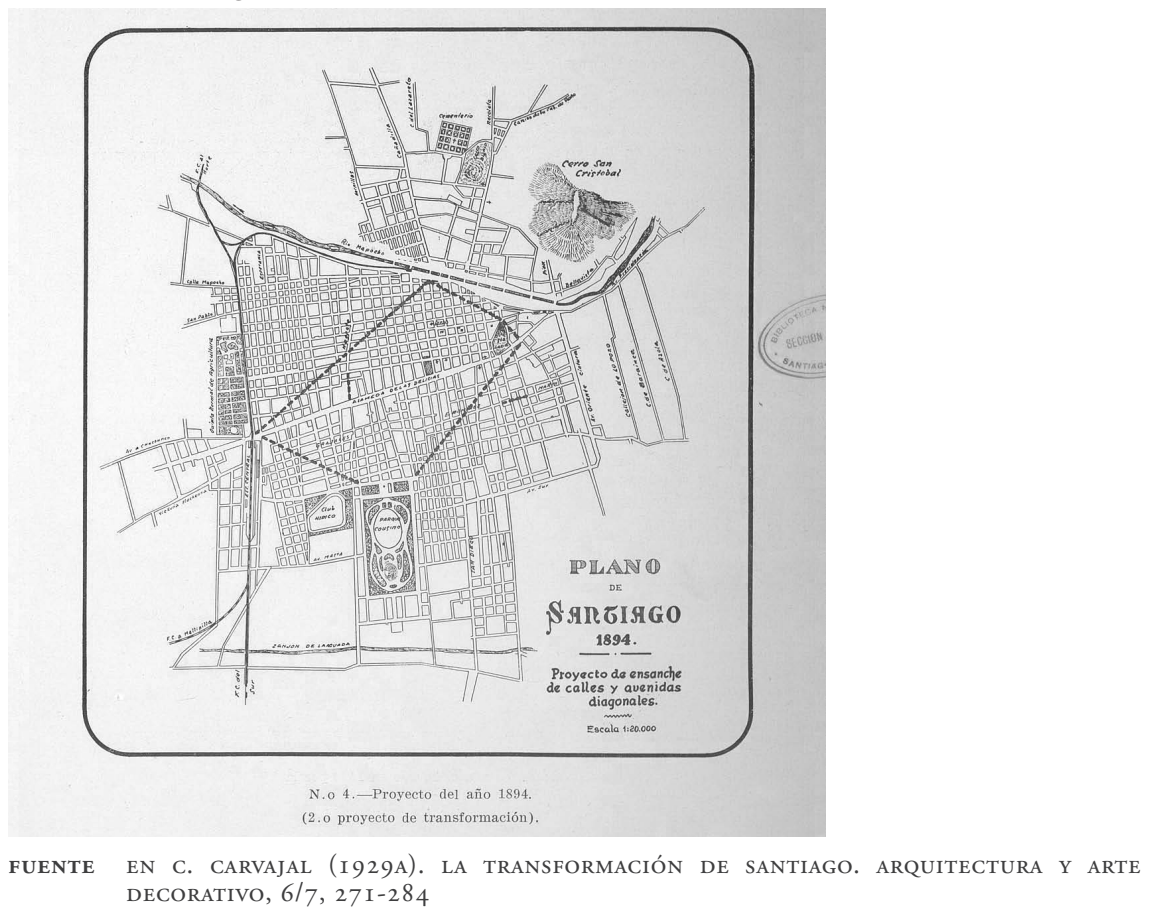

En el documento redactado por Bertrand (1895), este realizó importantes críticas al proyecto aprobado por la Cámara de Diputados. A su juicio, existían graves defectos en la aplicación de las líneas en el plano, que llevarían a que el gravamen fuese sumamente alto y pesara sobre la ciudad por largos ańos. Por otro lado, el proyecto no suministraría una comunicación rápida y cómoda para el tráfico de vehículos, situación que podría ser subsanada con menores costos a partir de un mayor uso del principio que se había utilizado en las transformaciones de las grandes ciudades modernas: diagonales para conectar de una manera expedita los centros de gran afluencia, como mercados, paseos y estaciones de ferrocarriles, con sectores más alejados.

Las diagonales fueron consideradas elementos importantes de modernización en los diferentes proyectos de transformación de las capitales latinoamericanas durante los años finales del siglo xix y las primeras décadas del xx (Almandoz, 2013). En este sentido, romper con la estructura colonial de damero tendría un impacto no solo práctico en la trama urbana, sino también un valor simbólico, al apreciarse de manera concreta cómo la modernidad irrumpía en la ciudad homogénea y tradicional (Mackenna, 1915; Valdés, 1914, 1922).

Respecto al informe realizado por Manuel Concha, este expresó que la Municipalidad pidió "un nuevo informe a la Direccion de Obras Municipales sobre la conveniencia que hai en aprobar el proyecto de la Ilustre Corporacion (...) i desvanecer la 
desfavorable impresion que pueden producir [las observaciones de A. Bertrand] en el ánimo de la Honorable Cámara de Senadores [sic]” (c.s., 28ª s.E., 1894, p. 491). Las críticas publicadas por Bertrand pusieron en peligro las posibilidades de aprobar definitivamente el proyecto en el Senado y, por tanto, de comenzar a llevar adelante las remodelaciones que eran consideradas por algunos como imprescindibles para mejorar no solo la calidad de vida de los habitantes de la capital, sino también la imagen que esta debía proyectar.

Con este objetivo presente, Manuel Concha destacó los beneficios que el proyecto presentaba. Puso énfasis en la higiene y la necesidad de crear anchas avenidas para resolver los problemas sanitarios de la ciudad. Por otra parte, y con el ancho de las calles que se proponía ${ }^{5}$, rebatía la idea de crear un gran número de diagonales, puesto que las avenidas conectarían perfectamente las calles y caminos de entrada a la ciudad con los centros de mayor movimiento. Finalmente, argumentó que las rentas de la Municipalidad de Santiago no se podían comparar con las de las grandes ciudades europeas, por lo que expropiar todos los terrenos que exigiría la creación de diagonales en la ciudad, sería impracticable (c.s., 28 a s.E., 1894).

Si bien las ideas presentadas por Manuel Concha eran menos ambiciosas que las propuestas por Bertrand, tendían a resguardar de mejor manera los costos de la intervención, sin transar el objetivo principal de modernizar la ciudad. En consideración a que ambos informes aportaban a la discusión, fueron adjuntados con los demás antecedentes del proyecto, volviendo a Comisión en 1898, sin que existiera certeza alguna respecto a la situación de las obras que en esos momentos se encontraban en ejecución (C.s., 53a s.E., 1898).

En efecto, la ciudad seguía creciendo. Nuevos edificios se construían y se aprobaba el ensanche de algunas calles. En este contexto surgían diversos problemas, no solo para la Municipalidad, sino también para los propietarios de terrenos y para el Fisco. Por una parte, la Municipalidad aprobaba proyectos de construcción y ensanche de calles siguiendo una línea arbitraria impuesta por sus empleados. Por otra, algunos propietarios preferían esperar a que se dictara una ley respecto a la línea definitiva de construcción, para no tener que demoler luego parte de sus edificaciones recién construidas. Finalmente, el Fisco también era perjudicado, pues no podía realizar ventas de terrenos, ya que para ello se debía dictar una ley que las autorizara, lo cual no podía llevarse a cabo sin la definición de las líneas de construcción (c.s., $8^{\text {a }}$ S.E., 1898). Ante este escenario, la redacción del informe de la Comisión de Gobierno se hacía urgente.

Un factor fundamental para comprender la demora en el despacho del informe es la inexistencia de una fórmula de clausura de los debates en el Congreso durante esos años, por lo que las intervenciones y discusiones se podían prolongar de manera indefinida. Por su parte, Patricio Gross $(1989,1990,1995)$ ha defendido en sus estudios que los intereses de los propietarios de las manzanas centrales de la capital habrían sido la mayor traba con que se habrían encontrado estos proyectos de transformación, ya que los estratos sociales más altos no habrían estado dispuestos a 
transar el derecho a la propiedad, lo que les garantizaba el control respecto a la entrada de estratos más bajos al centro de Santiago a partir de una mayor accesibilidad. Carlos Carvajal (1929a), refiriéndose a los proyectos presentados al Congreso en 1912, expresó de igual manera que su demora en la aprobación se debía a los "intereses creados" (p. 279). Así, se habrían disfrazado estos intereses particulares con argumentos sobre la escasez de fondos nacionales.

La espera por la aprobación del proyecto duró nueve años, hasta que en junio de 1907 el senador Ismael Valdés logró insertar el debate en torno a la transformación de la capital en las discusiones parlamentarias. No es casualidad que ese año se reabriera la discusión. Un año antes, el 16 de agosto de 1906, un gran terremoto había golpeado la zona central del país, destruyendo gran parte de Valparaíso. La ciudad de Santiago también se había visto afectada, quedando algunas edificaciones, sobre todo las más antiguas y de adobe, destruidas. En los meses posteriores al desastre se mostró una gran preocupación por reparar los daños más inmediatos, pero solo en los ańos siguientes comenzarían a ejecutarse las construcciones definitivas. Justamente en este punto radicó la principal preocupación de Valdés. Edificaciones sólidas comenzarían a construirse sin que aún existiera un plano aprobado en el que se establecieran las normas asociadas al proyecto de transformación de Santiago, "(...)porque hai una série de leyes que todas juntas no constituyen un plan de transformacion único [sic]" (c.s., 9a s.o., 1907, p. 184). La celebración del Centenario hacia 1910 también podría haber motivado la discusión, aunque para preparar las festividades y proyectar las construcciones que se inaugurarían ese año se había creado una comisión abocada únicamente a tales labores (Pérez, 2016; Serra, 2015).

El proyecto se resolvió finalmente en 1909. En agosto de ese año se nombró una Comisión Especial, cuyo encargo fue crear un informe que tuviera en consideración todos los antecedentes adjuntados al proyecto. Particular énfasis puso el Primer Alcalde de la Municipalidad de Santiago, Joaquín Marchant, en la conveniencia de llevar a cabo tanto el proyecto aprobado por la Cámara de Diputados en 1894, como los planteamientos expuestos por Alejandro Bertrand respecto a la creación de diagonales en la trama urbana. Ambos proyectos le parecían dignos de ser tomados en cuenta; señalaba al respecto que "si el primero, ademas de la mejor circulacion, contempla principalmente el ornato, hijiene i belleza de la ciudad, el segundo consulta la mejor viabilidad i el mas espedito i fácil tránsito en las calles [sic]" (c.s., 49a s.o., 1909, p. 844). De este modo, con la realización de ambos proyectos se lograría satisfacer los tres motores de la urbanística academicista: higiene urbana, sistemas de comunicación interna y el "embellecimiento" o "estética edilicia" (Fuentes, 2009).

Luego de diecisiete años desde el inicio de la discusión, el proyecto presentado por la Comisión se aprobó de manera expedita, siendo publicado oficialmente bajo la ley número 2203 el 16 de septiembre del mismo año. En ella se dejó la creación de diagonales para un nuevo proyecto y se estableció que el trazado de calles, plazas y avenidas estaría sujeto a un plano que debía ser aprobado por la Municipalidad. De este modo, el primer paso para poner en marcha la anhelada transformación de 
Santiago, como un proyecto global de intervención en la ciudad, quedó exclusivamente en manos de esta institución.

Resulta paradójico que precisamente aquello que se estableció como la solución definitiva para alcanzar el progreso urbano de la capital, terminara siendo su mayor piedra de tope, puesto que las autoridades municipales nunca aprobaron el plano conforme a la ley y, por tanto, el Municipio no pudo contar con las herramientas para regular el crecimiento urbano de Santiago conforme a un proyecto de ciudad futura. A raíz de que algunas edificaciones consideraron las nuevas líneas establecidas por ley, que instituía un ancho mínimo de 15 metros por igual para todas las calles de la ciudad sin jerarquías ni zonificaciones, y que algunas de las más antiguas sobresalían de ellas, esta ley fue denominada como "la ley del serrucho" (Carvajal, 1929a; Mackenna, 1915).

\section{El último impulso transformador del periodo, 1910-1929}

Para 1910 ya se identifican denuncias sobre las malas prácticas que trajo consigo la recientemente aprobada Ley 2203. Estas acusaciones abrirán un nuevo periodo de discusión en torno a la transformación de Santiago, a solo un ańo de promulgada dicha ley. Luego de la celebración del Centenario de la República, el senador Ramón Subercaseaux denunció que las Municipalidades se habían convertido en una especie de "cuarto poder", cometiendo abusos y actuando en contradicción con la ley. Desde 1891, con la aprobación de una ley comúnmente denominada Ley de Comuna Autónoma, que transfirió gran parte de las facultades ejercidas por la Administración Central a las Municipalidades, estas adquirieron una gran capacidad de gestión y de toma de decisiones (Salazar \& Pinto, 1999). En este contexto se pueden comprender las palabras de Subercaseaux, quien denunció a la Municipalidad de Santiago señalando que "no fija las líneas de construccion conforme a la lei, cuando los municipales o sus amigos se encuentran interesados en esto [sic]" (c.s., 16 a s.E., 1910, p. 310).

El evidente abuso ejercido por algunos empleados municipales, amparados por la inexistencia de un Plano Oficial de Transformación de Santiago, fue recurrente. Ante esta situación, los empleados municipales decidieron asumir la tarea de dar las líneas de construcción, tomando en cuenta -aunque no siempre- los trazos dibujados en el plano que poseían, pero que aún no había sido aprobado de manera oficial. Así, el plan de transformación urbana que tenía como base la regulación de apertura y trazado de calles, líneas y alturas de construcción, se vio frustrado en su ejecución, sin que las dificultades de las últimas décadas hubiesen sido superadas.

En el intento por buscar alguna manera expedita de salvar la situación, y gracias a la iniciativa de Ismael Valdés, en noviembre de 1911 se creó una Comisión Mixta, de senadores y diputados, con el fin de aprobar definitivamente el plano de transformación por medio de una ley (C.D., 28a s.E., 1911). La Comisión estudió el proyecto de aprobación del plano y además contó con diferentes informes remitidos al Congreso durante 1912. Posiblemente sea este el caso en el que mejor se puede apreciar el gran interés público que por esos años generó la discusión, y que 
incentivó a que nuevos actores, como la Sociedad Central de Arquitectos [sca] y la Comisión de Bellas Artes, participaran en el debate.

A partir de la experiencia de las últimas décadas y de las dos leyes de transformación aprobadas con anterioridad, en el informe presentado por la Comisión Mixta a la Cámara de Diputados se incluyen con mayor detalle los fondos para el pago de las expropiaciones necesarias, se fijan claramente los límites de la ciudad (figura 3) y se crea una entidad permanente a cargo de la dirección, inspección y administración de las obras, denominada Junta de Transformación. Esta última incorporación al proyecto resulta notable, pues buscaba reemplazar completamente las labores que se le habían asignado a la Municipalidad en la ley de 1909 en cuanto a dirección y vigilancia de las obras, por una Junta permanente que tuviese la transformación de Santiago como única responsabilidad, dando cuenta del fracaso municipal en la administración de la trasformación de la capital.

Figura 3 | Plano Oficial. Proyecto de transformación acordado por la $\mathrm{H}$. Comisión de Senadores y Diputados (1912) por Luis Felipe Laso

\section{PLANO OFICIAL}

Proyecto de la trasformacion acordado por la H. Comision de Senadores y Diputados

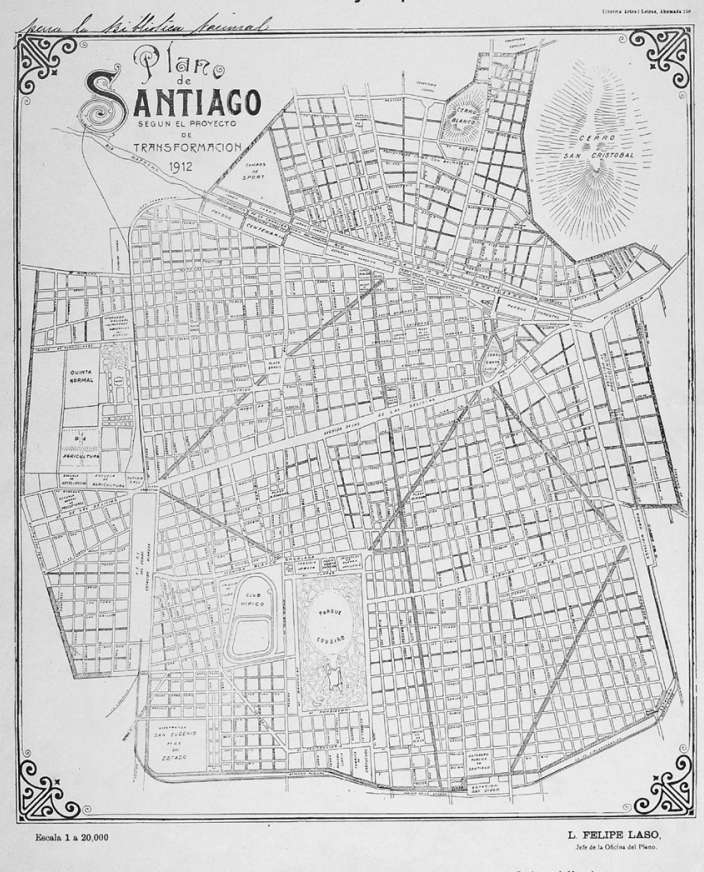

FUENTE COLECCIONES BIBLIOTECA NACIONAL DE CHILE. ARCHIVO VISUAL DE SANTIAGO. HTTP:// WWW.ARCHIVOVISUAL.CL/PLANO-DE-SANTIAGO-SEGUN-EL-PROYECTO-DE-TRANSFORMACION 
En el segundo informe presentado al Congreso se aprecia por primera vez la participación activa de la Sociedad Central de Arquitectos en la discusión parlamentaria. Para la Sociedad, creada en 1907 (Aguirre, 2011), los estudios profesionales y la práctica de sus miembros les permitía expresar opiniones e ideas sobre el tema en cuestión, así como cooperar en la elaboración de la ley (c.D., 21 a s.o., 1912). A grandes rasgos, los arquitectos propusieron la creación de diversas diagonales que conectarían el Barrio Cívico con algunos puntos relevantes de la ciudad y zonas alejadas, además de una vía de circunvalación que estableciera los límites urbanos (figura 4).

\section{FIgURA 4 Plano de Santiago con el proyecto de transformación de la Sociedad Central de Arquitectos. Trazado definitivo (1912) por Carlos Carvajal}

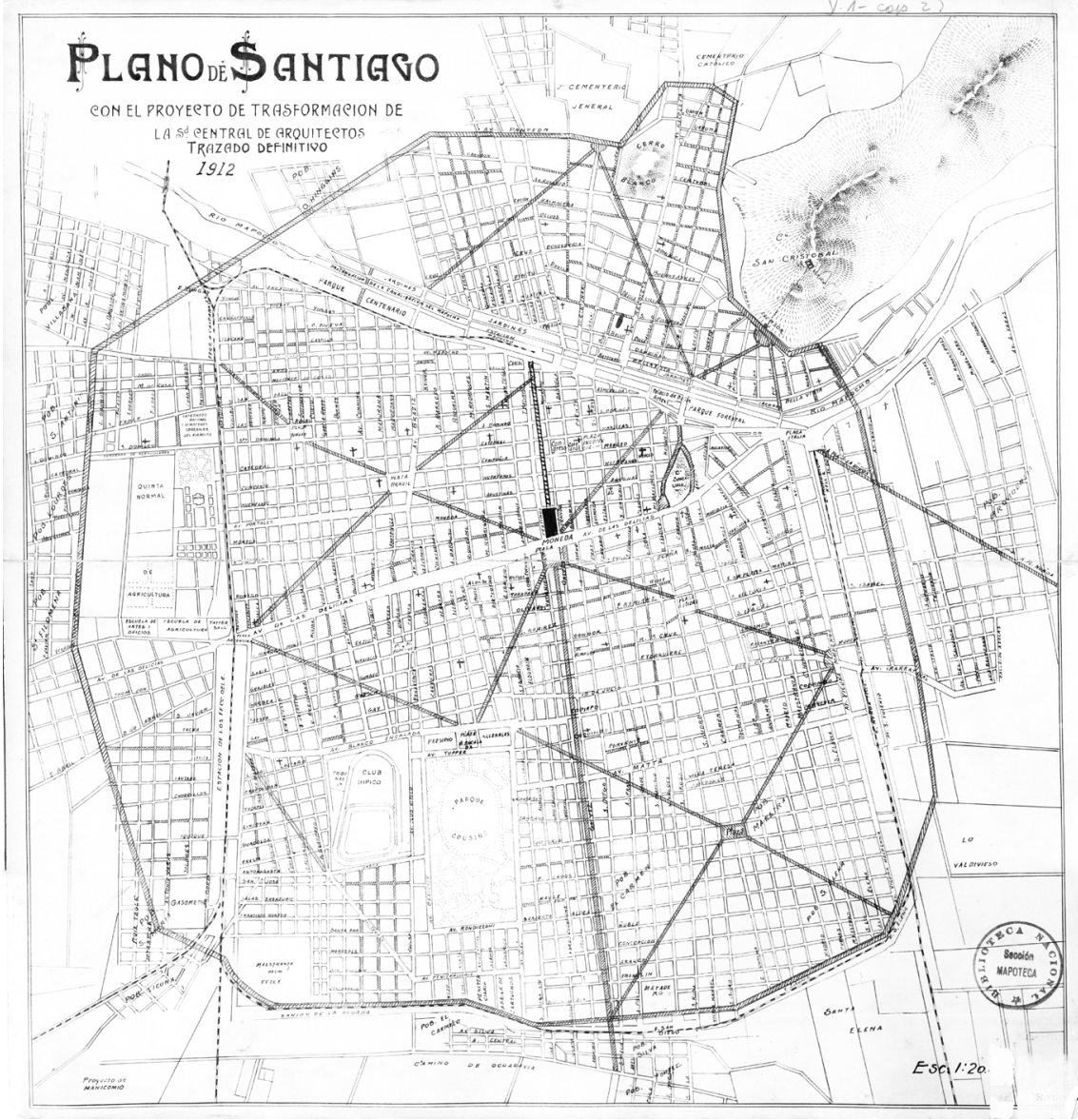

FUENTE COLECCIONES BIBLIOTECA NACIONAL DE CHILE. ARCHIVO VISUAL DE SANTIAGO. HTTP://WWW. ARCHIVOVISUAL.CL/PLANO-DE-SANTIAGO\%C2\%AOCON-EL-PROYECTO-DE-TRANSFORMACION 
Respecto a la creación de la Junta de Transformación, se encontraban de acuerdo con ella, pero brindándole facultades más amplias y cambiándole el nombre a Consejo Permanente de los Servicios de Arquitectura de Santiago. Este Consejo, además de cumplir las funciones propuestas por la Comisión Mixta, debía ocuparse "de todo lo que tiene relacion con el progreso estético de la ciudad, de ornato i de embellecimiento [sic]" (C.D., 21 a s.O., 1912, p. 687). Siguiendo los preceptos del urbanismo moderno, particularmente con el uso de diagonales y la anchura de las calles presentes en su proyecto, la SCA lograba unir en una sola propuesta la necesidad de conectividad y fluidez en la circulación de vehículos con las ideas de embellecimiento y ornato, que se podían estimar, según Carlos Carvajal (1929a), no solo de interés local, sino también de dignidad nacional.

El plano presentado por la sca fue creado en colaboración con el Instituto de Ingenieros y la Comisión de Bellas Artes. Esta última también remitió un informe al Congreso Nacional, en el que indicaba que, a su parecer, el proyecto de la sca satisfacía las necesidades de ese momento y futuras de la capital, de mejor manera que el proyecto presentado por la Comisión Mixta. Resulta interesante la manera en que se presenta el trabajo conjunto de las tres instituciones mencionadas, puesto que ellas se establecieron como autoridades técnicas competentes para aportar en la discusión de la transformación de la capital. En efecto, Alberto Mackenna, en su texto Santiago futuro (1915), en el que se recogen algunas de las conferencias presentadas en la Biblioteca Nacional en torno a la transformación de Santiago, exponía: "El sentimiento del artista se ha unido al criterio de economista y al espíritu de previsión social para confeccionar un plano en el cual están consultadas todas las exigencias de la edilidad moderna" (p. 60). Desde la aparición del plano de Ernest Ansart en 1875, todas las propuestas habían consultado la experticia técnica de ingenieros, siendo esta la primera vez que se observa la incorporación de los arquitectos como gremio en la articulación, no solo de un plano, sino de un proyecto de transformación de la ciudad.

Un último informe redactado en el marco de esta discusión fue emitido por una Comisión Especial formada en la Cámara de Diputados. Al existir una gran cantidad de informes, en julio de 1912 se creó una Comisión que, tomando en cuenta todos los documentos expuestos, pudiera redactar un informe único (C.D., 29a s.o., 1912). En él se proponía a la Cámara que aprobase el proyecto presentado por la Comisión Mixta con algunas modificaciones, entre las cuales la más urgente era la derogación de la ley de 1909, ya que la Municipalidad de Santiago todavía no aprobaba el plano de transformación. De este modo, aún no existía claridad sobre qué decisión tomar en ciertos casos de construcción o reconstrucción, lo que, sumado a la apertura y ensanche de algunas calles, llevó por esos años a una renovación desarticulada de la ciudad.

Durante este periodo, el contexto internacional era poco alentador, sobre todo hacia 1914. Si bien la economía nacional se habría visto afectada tras el inicio de la Gran Guerra, en los años siguientes lograría sostenerse debido al desarrollo de una economía volcada a la exportación de salitre (Bethell, 2000; Cariola \& Sunkel, 1991; Ortega, 2012). 
Para 1915, el diálogo no prosperaba en las salas del Congreso y, por tanto, no se tomaba ninguna medida tendiente a mejorar la situación de la capital (c.s., 20a s.E., 1915). Ante esta situación, el Gobierno decidió nombrar una nueva Comisión formada por parlamentarios y otras autoridades locales, cuya tarea sería "presentar al Gobierno un proyecto de lei tendiente a la modernizacion de la ciudad i a su ensanche futuro... [sic]" (C.D., 32a s.E., 1918, p. 951). En el periodo que comprende este estudio, este intento de dinamizar la discusión, representa un último esfuerzo por concretar la anhelada transformación de Santiago, a través de la cual se buscaba generar un plan de mejoramiento integral de la ciudad que, inspirada en los trabajos haussmannianos de París, estableciera los principales lineamientos a los que debía apuntar cualquier futura intervención.

La propuesta presentada por la Comisión abandona la idea de ensanchar uniformemente las calles de la ciudad, optando por definir un ancho mínimo solo en las nuevas avenidas, lo que permitiría en un par de años, y a un menor costo, "una transformación que consulte todas las condiciones de una ciudad moderna, aunque la mayor parte de las calles conserven su actual anchura [sic]" (C.D., 32 a s.E., 1918, p. 851). Por otra parte, se suprime la formación de barrios por medio de la especulación, en un intento de descartar los abusos que por ańos fueron cometidos por esa vía. El Presidente de la República debía aprobar el plano y ordenar su revisión cada quince años, "con el objeto de proponer las reformas que la práctica i nuevas circunstancias aconsejaren [sic]” (p. 851). La Junta de Transformación supliría totalmente las labores que se le habían asignado a la Municipalidad en la ley de 1909 en cuanto a dirección y vigilancia de las obras, legando la ejecución de las obras a la Oficina del Plano, dependiente del Presidente de la República. De esta manera, se concibió una administración sumamente centralizada de la transformación de la ciudad.

Al ser recibido en la Cámara de Diputados en 1918, el proyecto se envió a Comisión para su análisis y evaluación, donde encontraría el fin de su recorrido, puesto que ahí quedó archivado. No se encuentran registros en las discusiones parlamentarias de que se volviera a tratar entre 1918 y 1929 . A pesar de que ya no se debatirá sobre un proyecto de intervención de Santiago a gran escala, sí estarán dentro de las tablas de discusión del Congreso Nacional proyectos específicos como el de alcantarillado, pavimentación o apertura de algunas avenidas diagonales.

El contexto al que se enfrentaría este proyecto en la década de 1920 le podría haber impedido seguir su curso. Por una parte, el gobierno de Arturo Alessandri Palma tuvo que hacer frente a diversas dificultades políticas, y sobre todo sociales, expresadas particularmente en la llamada "cuestión social" (Grez, 1995). Por su parte, la clausura del Congreso Nacional desde el 12 de septiembre de 1924 hasta 1925 por la Junta Militar impidió por un tiempo llevar adelante cualquier discusión. Una de las consecuencias de la intervención militar fue la disolución de la Municipalidad de Santiago, así como la de Valparaíso, el 26 de septiembre de 1924, y la implantación de un nuevo sistema de gobierno local: las Juntas de Vecinos. Estas fueron elegidas por decreto ministerial y solo se designó a un alcalde para cada comuna (León, 1975; Salazar \& Pinto, 1999; Walter, 2005).

Sin embargo, se debe mencionar la existencia de dos propuestas de Transformación de Santiago presentadas al Congreso en 1925, pero sobre las cuales pareciera 
no haber existido mayor discusión o resolución. Una de ellas es el proyecto aprobado por la Junta de Vecinos, bajo el mandato del alcalde de Santiago entre 1925 y 1927, Luis Phillips (Carvajal, 1929b; Pavez, 2000; Sección Técnica, 1926). La otra es un Decreto de Ley presentado el mismo año por Francisco Mardones (1925), quien ejerció como Ministro de Obras Públicas y posteriormente como Ministro del Interior en 1925 (Pinto, 1991).

El 30 de enero de 1929 se promulgó la Ley 4563, que dispuso que las ciudades que contaban con más de veinte mil habitantes enviaran un anteproyecto de transformación que el Presidente de la República debería ordenar como definitivo. La publicación de esta ley significó el fin de la discusión parlamentaria específica sobre la transformación de Santiago en este periodo. En 1930 se comienza a aplicar una manera distinta de comprender la intervención en la ciudad, a partir de una nueva práctica científica del urbanismo (Hofer, 2006). Este enfoque será visible en la realización del Barrio Cívico y en la proyección de un plan de urbanización para Santiago basado en las ideas del urbanista austríaco Karl Brunner (Rosas, Hidalgo, Strabucchi \& Bannen, 2015). La nueva forma de concebir la ciudad se concretó con la aprobación de la primera ley sobre urbanización y construcciones en 1929 que, tomando en cuenta algunos aspectos regulatorios de la última discusión, contempló dentro de su texto la construcción antisísmica tras el terremoto de Talca de 1928 (Munizaga, 2014). Lo anterior fue reforzado en 1931 con el Decreto con Fuerza de Ley número 345, que aprobó la Ley y Ordenanza General sobre Construcciones y Urbanización. De este modo, a partir de la década de 1930 se establecerá una manera diferente de enfrentar y proyectar los problemas de la ciudad (Fuentes, 2009; Gurovich, 2003).

\section{Consideraciones finales}

A través de la revisión de la discusión sobre la transformación de Santiago en el Congreso Nacional entre 1872 y 1929 , es posible apreciar que las principales preocupaciones urbanas, surgidas a partir de los proyectos que buscaban generar una amplia intervención en la ciudad, se insertan en momentos y contextos específicos. Se observan ciertas preocupaciones transversales a todo el periodo analizado, que se relacionan con la vialidad, salubridad e higiene; y con la intención de modernizar la ciudad, establecer líneas generales de crecimiento y planificación urbana, normar la construcción de edificaciones, ampliar y normar los servicios básicos, y la construcción de nuevos barrios. Todo ello con claras miras a las intervenciones urbanas llevadas adelante en las grandes capitales europeas durante la segunda mitad del siglo XIX.

Asimismo, se aprecian diversas problemáticas que estarán presentes a lo largo de este periodo, como la falta de recursos disponibles para intervenir la ciudad en los puntos que los proyectos indicaban originalmente y que efectivamente proponían una intervención a gran escala de la capital. A pesar de que algunas mejoras se pensaron para ser desarrolladas en un plazo de varios años, hubo otras preocupaciones relacionadas con el acontecer nacional que merecieron mayor prioridad, así como la presencia de intereses privados de propietarios de inmuebles en el área 
central que habrían trabado la discusión parlamentaria en algunos momentos. Por otra parte, las contingencias del contexto político, social y económico impidieron que se desarrollara una discusión expedita y se lograra una continuidad entre las propuestas.

No obstante estos contratiempos, los factores que realmente impidieron que se lograra una verdadera transformación de la ciudad de Santiago se relacionan más bien con las consecuencias de las dos leyes aprobadas, una en 1874 y la otra en 1909. Si en el primer caso se hubiese establecido claramente una sanción asociada al incumplimiento de la ley, y en el segundo la Municipalidad de Santiago hubiese aprobado inmediatamente el plano de manera oficial, gran parte de las consecuencias negativas, que se concretaron en el incumplimiento de ambas leyes por parte de propietarios y autoridades municipales, podrían haber sido evitadas.

La falta de fondos impidió que se aprobase un proyecto de intervención ambicioso, como algunos propusieron en los debates parlamentarios, por lo que la decisión que se tomó fue la de aprobar los proyectos que permitieran mejorar las condiciones urbanas de la capital en la medida de lo posible, intentando conciliar las expectativas con las posibilidades reales de llevar adelante un extendido plan de obras. No obstante, y en definitiva, la incapacidad de las autoridades para revertir rápidamente aquellas situaciones que ponían en peligro los objetivos de las leyes de 1874 y de 1909, impidió concretar una transformación urbana a gran escala en Santiago de Chile durante las décadas finales del siglo xIx y las primeras del siglo xx.

\section{Referencias bibliográficas}

Aguirre, B. \& Castillo, S. (2004). De la "gran aldea" a la ciudad de masas: El espacio público en Santiago de Chile, 1910-1929. Santiago: LOM.

Aguirre, M. (2011). Un triángulo estratégico para una inserción cultural. Organización gremial, revistas de arquitectura y modernidad 1907-1942. Revista de Arquitectura, 17(23), 1419. https://doi.org/10.5354/0719-5427.2013.26893

Almandoz, A. (ed.). (2010). Planning Latin America's capital cities, $1850-1950$ (2 $\left.2^{\text {nd }} e d.\right)$. Abingdon, Oxon: Routledge.

Almandoz, A. (2013). Modernización urbana en América Latina. De las grandes aldeas a las metrópolis masificadas. Santiago, Chile: Colección Estudios Urbanos uc / Instituto de Estudios Urbanos y Territoriales, Pontificia Universidad Católica de Chile.

Bethell, L. (ed.). (2000). Historia de América Latina (Vol. 7). Barcelona: Crítica.

Bertrand, A. (1895). Observaciones sobre el actual proyecto de transformación de Santiago. En Direccion Jeneral de Obras Publicas, Proyecto de transformación de los barrios vecinos al Canal del Mapocho. Presentado al Consejo de Obras Públicas por la Direccion Jeneral del Ramo (pp. 22-27). Santiago: Imprenta Cervantes.

Boletines de las Sesiones Ordinarias y Extraordinarias de la Cámara de Diputados, 1872-1929. Santiago: Imprenta Nacional.

Boletines de las Sesiones Ordinarias y Extraordinarias de la Cámara de Senadores 1872-1929. Santiago: Imprenta Nacional. 
Cariola, C. \& Sunkel, O. (1991). Un siglo de historia económica de Chile, 1830-1930. Santiago: Universitaria.

Carvajal, C. (1929a). La transformación de Santiago. Arquitectura y Arte Decorativo, 1(6/7), 271-284. En http://www.memoriachilena.cl/602/w3-article-318082.html

Carvajal, C. (1929b). La transformación de Santiago (Continuación). Arquitectura y Arte Decorativo, 1(8), 339-348. En http://www.memoriachilena.cl/602/w3-article-318083. html

Collier, S. \& Sater, W. (1998). Historia de Chile 1808-1994. Madrid: Cambridge University Press.

DeJean, J. (2014). How Paris became Paris. The invention of the modern city. Nueva York: Bloomsbury.

De Ramón, A. (1985). Estudio de una periferia urbana. Santiago de Chile 1850-1900. Historia, (38), 199-289. En https://repositorio.uc.cl/handle/11534/9656

Fernández, E. (2014). La transformación urbana de Santiago de Chile: finanzas, obras públicas y discurso político (1870-1910). Amérique Latine Histoire et Mémoire. Les Cabiers ALHIM, (28). En http://journals.openedition.org/alhim/5091

Folchi, M. (2007). La higiene, la salubridad pública y el problema de la vivienda popular en Santiago de Chile, 1843-1925. En L. López (coord.), Perfiles habitacionales y condiciones ambientales: historia urbana de Latinoamérica siglos XVII-XX (pp. 361-388). Puebla: Benemérita Universidad Autónoma de Puebla. En http://repositorio.uchile.cl/ handle/2250/122785

Fuentes Hernández, P. (2009). Antecedentes de la arquitectura moderna en Chile 1894-1929. Concepción: Universidad del Bío-Bío.

Gazmuri, C. (2012). Historia de Chile 1891-1994. Politica, economía, sociedad, cultura, vida privada, episodios. Santiago: RIL /Instituto de Historia, Pontificia Universidad Católica de Chile.

Giedion, S. (2009). Espacio, tiempo y arquitectura. Barcelona: Reverté.

Gorelik, A. (2003). Ciudad, modernidad, modernización. Universitas Humanistica, 56(56), 11-27. En http://revistas.javeriana.edu.co/index.php/univhumanistica/article/ view/9694/7926

Gravagnuolo, B. (2010). Historia del urbanismo en Europa 1750-1960 (2a reimp.). Madrid: Akal.

Grez, S. (recop.). (1995). La "cuestión social" en Chile. Ideas y debates precursores (1804-1902). Fuentes para la historia de la República (Vol. vII). Santiago: Ediciones de la Dirección de Bibliotecas, Archivos y Museos.

Gross, P. (1989). Un acercamiento a los planes de transformación de Santiago de Chile (18751985). En J. Hardoy \& P. Morse (comps.). Nuevas perspectiva en los estudios sobe historia urbana latinoamericana (pp. 305-325). Buenos Aires: Grupo Editor Latinoamericano S.R.L.

Gross, P. (1990). Santiago de Chile: ideología y modelos urbanos. EURE, 16(48), 67-85. En http://www.eure.cl/index.php/eure/article/view/1026

Gross, P. (1995). Utopías haussmannianas y planes de transformación 1894-1925. En P. Bannen (ed.), Santiago de Chile. Quince escritos y cien imágenes. Santiago: ARQ. 
Gurovich, A. (2000). Conflictos y negociaciones: La planificación urbana en el desarrollo del Gran Santiago, Chile. Revista de Urbanismo, (2), 1-39. https://doi.org/10.5354/07175051.2011.12304

Gurovich, A. (2003). La solitaria estrella: en torno a la realización del Barrio Cívico de Santiago de Chile, 1846-1946. Revista de Urbanismo, (7), 1-28. https://doi.org/10.5354/07175051.2003.6214

Harvey, D. (2003). Paris, capital of modernity. Nueva York \& Londres: Routledge.

Heise, J. (1974). Historia de Chile. El periodo parlamentario 1861-1925. Santiago: Andrés Bello. Hidalgo, R. (2002). Vivienda social y espacio urbano en Santiago de Chile. Una mirada retrospectiva a la acción del Estado en las primeras décadas del Siglo xx. EURE, 28(83), 83-106. https://doi.org/10.4067/S0250-71612002008300006

Hidalgo, R. \& Camus, P. (2006). La difusión de las ideas urbanísticas modernas en Chile desde la transformación de ciudades a la ciudad lineal. En J. Valenzuela (ed.), Historias urbanas. Homenaje a Armando de Ramón (pp. 241-262). Santiago: Ediciones Universidad Católica.

Hidalgo, R. \& Sánchez, R. (2006). La ciudad con ojos de autoridad. El plan de reforma de Santiago del intendente José Miguel de la Barra 1843-1849. Scripta Nova, 10(218/31). En http://www.ub.edu/geocrit/sn/sn-218-31.htm

Hofer, A. (2003). Karl Brunner y el urbanismo europeo en América Latina. Bogotá: El Áncora.

Hofer, A. (2006). El origen de la metrópolis. Las propuestas de Karl Brunner. En Ilustre Municipalidad de Santiago, Dirección de Obras Municipales (ed.), Santiago Centro. Un siglo de transformaciones (pp. 28-37) Santiago: Ilustre Municipalidad de Santiago.

Ibarra, M. (2016). Hygiene and public health in Santiago de Chile's urban agenda, 1892-1927. Planning Perspectives, 31(2), 181-203. https://doi.org/10.1080/02665433.2015.107 0280

Larraín, J. (1997). La trayectoria latinoamericana a la modernidad. Estudios Públicos, (66), 313333. En https://www.cepchile.cl/la-trayectoria-latinoamericana-a-la-modernidad/ cep/2016-03-03/184857.html

León Echaiz, R. (1975). Historia de Santiago (Vol. 2). Santiago: I. Municipalidad de Santiago.

Levin, M., Forgan, S., Hessler, M., Kargon, R. \& Low, M. (2010). Urban modernity. Cultural innovation in the second industrial revolution. Cambridge, MA: The MIT Press. https:// doi.org/10.7551/mitpress/9780262013987.001.0001

Mackenna, A. (1915). Santiago futuro. Conferencias sobre los proyectos de transformación de Santiago. Santiago / Valparaíso: Soc. Imprenta-Litografía Barcelona.

Mardones, F. (1925, septiembre). Proyecto de ley de transformación de Santiago. Anales del Instituto de Ingenieros de Chile, 10(26), 505-522. En http://www.nuevosfoliosbioetica. uchile.cl/index.php/AICH/article/view/34641/36343

Martínez, R. (2006). Santiago: Los planos de transformación 1894-1928. En Ilustre Municipalidad de Santiago, Dirección de Obras Municipales (ed.), Santiago Centro. Un siglo de transformaciones (pp. 22-27). Santiago: Ilustre Municipalidad de Santiago.

Martínez, R. (2007a). Santiago de Chile: los planos de su historia. Siglos XVI a XX: de aldea a metrópolis. Santiago: Facultad de Arquitectura, Urbanismo y Paisaje, Universidad Central de Chile.

Martínez, R. (2007b). Santiago, los planos de transformación 1894-1929. Revista Electrónica DUßP, 4(10). En http://dup.ucentral.cl/10_santiago_planos.htm 
Munizaga, G. (2014). Diseño urbano: teoría y método (3a ed.). Santiago: Ediciones Universidad Católica.

Ortega, L. (2010). La política, las finanzas públicas y la construcción territorial. Chile 18301887. Ensayo de interpretación. Universum, 25(1), 140-150. En http://universum. utalca.cl/contenido/index-10-1/ortega.html

Ortega, L. (2012). La crisis de 1914-1924 y el sector fabril en Chile. Historia, 45(2), 433-454. https://doi.org/10.4067/S0717-71942012000200003

Pavez, M. I. (2000). El Plan Brunner para Santiago: potencial de desarrollo de las totalidades, subtotalidades y fragmentos viales en la comuna de Independencia. Revista de Urbanismo, (3), 1-40. https://doi.org/10.5354/0717-5051.2011.11786

Pérez, F. (2016). Arquitectura en el Chile del siglo xx. Vol. I: Iniciando el nuevo siglo 1890-1930. Santiago: ARQ.

Pérez, F. \& Rosas, J. (2010). Cities within the city: Urban and architectural transfers in Santiago de Chile, 1840-1940. En A. Almandoz (ed.), Planning Latin America's capital cities 1850-1950 (cap. 5, pp. 109-138). Abingdon, Oxon: Routledge.

Pinto, F. (1991). Balmaceda y los gobiernos seudo-parlamentarios. Santiago: Andrés Bello.

Romero, J. L. (2014). Latinoamérica, las ciudades y las ideas (3a ed.). Buenos Aires: Siglo Veintiuno.

Rosas, J. (1985). La partición de la manzana. Cómo se modernizó Santiago de Chile. UR: Urbanismo Revista, (3), 29-38. En http://hdl.handle.net/2099/2992

Rosas, J., Hidalgo, G., Strabucchi, W. \& Bannen,P. (2015). La idea de "ciudad moderna" de Karl Brunner en tres líneas: el Plano Oficial de Urbanización de la comuna de Santiago, de 1939. Revista 180, (35), 10-17. En http://www.revista180.udp.cl/index. $\mathrm{php} /$ revista180/article/view/29/27

Salazar, G. \& Pinto, J. (1999). Historia contemporánea de Chile (Tomo I). Santiago: LOM.

Serra, D. (2015). ¿Celebrar o no celebrar? La organización de los festejos oficiales del centenario de la independencia de Chile, 1904-1910. Historia, 48(2), 595-626. https://doi. org/10.4067/S0717-7194201500020000

Strabucchi, W., Vicuña, M., Hidalgo, G. \& Rosas, J. (2013). El plano detallado de Santiago de Alejandro Bertrand (1889-1890). ARQ, (85), 66-81. https://doi.org/10.4067/S071769962013000300011

Valdés, I. (1906). Prácticas parlamentarias. Santiago: Imprenta Litografia i Encuadernacion Barcelona.

Valdés, I. (1914). La modernización de las ciudades y la transformación de Santiago. Conferencia dictada en la Biblioteca Nacional. Santiago: Imprenta La Ilustración.

Valdés, I. (1922, mayo). Conferencias sobre transformación de ciudades. Revista de Arquitectura, (1), 3-5.

Valdés Cange, J. (1910). Chile intimo en 1910. Santiago: Imprenta Universitaria.

Vicuńa, B. (1873). La transformación de Santiago. Santiago: Imprenta de la Librería del Mercurio.

Vicuña, M. (1996). El París americano: la oligarquía chilena como actor urbano en el siglo XIX. Santiago: Universidad Finis Terrae / Museo Histórico Nacional.

Walter, R. (2005). Urban growth in Santiago, Chile, 1891-1941. Redwood City, CA: Stanford University Press. 


\section{Leyes citadas}

Decreto con Fuerza de Ley 345 (1931). Ministerio del Interior. Sin título [Aprueba la Ley y Ordenanza General sobre Construcciones y Urbanización]. Promulgado el 20 de mayo de 1931. En http://bcn.cl/1vcux

Ley 2203 (1909). Ministerio del Interior. Fija las disposiciones a que deberá sujetarse la construccion de edificios, apertura, ensanche, union, prolongacion o rectificacion de calles de la ciudad de Santiago. Promulgada el 7 de septiembre de 1909. En http:// bcn.cl/241cs

Ley 4563 (1929). Ministerio del Interior. Sin título. [Sobre normas para la construcción de edificios y otras obras]. Promulgada el 30 de enero de 1929. En http://bcn.cl/1wngm

Ley s/n (1874). Organismo no especificado. Apertura i prolongacion de calles i paseos públicos en la ciudad de Santiago. Promulgada el 25 de junio de 1874. En http://bcn.cl/244e0

Ley s/n (1891).Ministerio del Interior. Proyecto de lei sobre organizacion i atribuciones de las municipalidades [Ley de Comuna Autónoma]. Promulgada el 22 de diciembre de 1981. http://bcn.cl/1x0au 